\title{
Surface structure of polymers and their model compounds observed by atomic force microscopy
}

\author{
W. Stocker, B. Bickmann, S.N. Magonov, H.-J. Cantow \\ Freiburger Material-Forschungszentrum F.M.F. and Institut für Makromolekulare Chemie, Albert-Ludwigs-Universität, \\ Stefan-Meier-Strasse 31A, W-7800 Freiburg, Germany \\ B. Lotz, J.-C. Wittmann \\ Institut Charles Sadron (CRM-EAHP), CNRS Université Louis Pasteur, 6 rue Boussingault, F-67083 Strasbourg Cedex, France
}

and

M. Möller

Department of Chemical Technology, University of Twente, P.O. Box 217, NL-7500 AE Enschede, The Netherlands

Received 12 August 1991

\begin{abstract}
Results of atomic force microscopy (AFM) of normal alkanes, polyethylene, isotactic polypropylene and of a diblock copolymer are presented. Various types of surfaces - naturally and epitaxially grown on different substrates - have been examined from hundreds of nanometers down to the atomic scale. Surface morphology and molecular arrangement have been visualized in AFM images. Atomic-scale AFM images with some defects have been observed on lamellar surfaces of normal alkanes $\mathrm{C}_{33} \mathrm{H}_{68}$. Arrays of oriented polyethylene molecules have been revealed at the surface of thin polyethylene films, epitaxially crystallized on the (001) face of an anthracene single crystal. Contact faces of thin films of isotactic polypropylene (iPP), epitaxially crystallized on various substrates (i.e. benzoic acid, potassium salt of 4-chloro-benzoic acid or polyamide 11), show the lamellar structure as well as the methyl side-group pattern in the exposed iPP (010) planes. AFM offers the way to distinguish the crystalline modification as well as the molecular conformation (left- and right-handed helices). Also the morphology of a microphase-separated block copolymer, polystyrene-b-poly-(2-vinylpyridine) (PS /PVP), has been observed by AFM.
\end{abstract}

\section{Introduction}

The invention of the atomic force microscope (AFM) has provided new opportunities for studying surfaces of non-conductive materials. The possibility of obtaining three-dimensional highresolution images under ambient conditions makes AFM an interesting technique for polymer structure investigations. It will be important if AFM can yield new detailed information about the conformation of macromolecules on polymer surfaces. At present, the main knowledge about molecular conformation (folds between adjacent crystalline stems and interlamellar links between adjacent lamellae) is based on studies in bulk crystallized samples [1]. Recently, normal alkanes and cyclic alkanes have been rediscovered as well defined model systems for the investigation of the crystallization of polyethylene [2]. Linear alkanes form extended-chain crystals with structures similar to polyethylene crystals. Due to their cyclic structure, cycloalkanes are forced to form lamel- 
lar crystals in which the adjacent stems are connected by tight folds. In first steps of AFM applications to polymers we have examined monocrystals of linear and cyclic alkanes [3].

In the present communication, the potential and the advantages of the AFM for morphology studies on different polymer samples are demonstrated. Flat surfaces of polymers on different substrates are well suited for AFM examination. Morphology patterns and molecular order on epitaxially crystallized polyethylene (PE) and isotactic polypropylene (iPP) will be discussed below. The samples of epitaxial crystallized iPP have been studied in more detail. Two different crystalline modifications, the monoclinic $\alpha$-phase and the triclinic $\gamma$-phase [4,5], were observed for iPP. Both structures are based on the same $3_{1}$ helical conformation of the chain [6]. AFM images of the monoclinic $\alpha$-phase, epitaxially crystallized on benzoic acid, have been published before in ref. [7]. AFM images of surfaces of $\gamma$-phase crystals of iPP, epitaxially crystallized on different substrates (benzoic acid, potassium salt of 4-chlorobenzoic acid and polyamide 11), will be discussed.

Worthwhile to note is that not only crystalline polymers can be studied by AFM. As an example, the AFM observations of a glassy block copolymer (PS/PYP) are presented.

\section{Experiment}

Low-molecular-weight iPP was produced by thermal chain scission of high-molecular-weight iPP under vacuum and vaporization on glass cover slides [8]. The product was comelted with the substrate between cover slides and slowly crystallized in a temperature gradient. After removal of the substrate by dissolution in hot methanol, the contact surface was ready for AFM examination. iPP was also crystallized on polyamide 11 (PA11), which was oriented by epitaxial crystallization. The free surface, naturally grown on PA11, was examined.

Polyethylene was epitaxially crystallized on anthracene as described in ref. [9].

An important requirement for samples to be studied by AFM is flatness of the surface.
Monocrystals of linear alkanes have a platelet shape and can be grown to relatively large size. The large lamellar surfaces are suitable for AFM studies. Monocrystals of orthorhombic $n$-tritriacontane $\mathrm{C}_{33} \mathrm{H}_{68}$ were prepared as described elsewhere [10].

The block copolymer PS / PVP was synthesized by sequential polymerization of styrene and 2 vinylpyridine in toluene with butyllithium as the initiator [11]. The molecular weight of the sample is $M_{\mathrm{n}}=40000$ and $M_{\mathrm{w}} / M_{\mathrm{n}}=1.08$ and the composition of the diblock PS/PVP is $4: 1$ and $6: 1$. Bulk materials were prepared by slow solvent evaporation of solutions of the polymer in $\mathrm{N}, \mathrm{N}$ dimethylformamide (5\% PS /PVP). AFM samples of PS/PVP were prepared by ultramicrotomy with a diamond knife. The second sample of this polymer was prepared by slow solvent evaporation. A $0.1 \%$ solution of the copolymer in toluene/methanole $(4: 1 \mathrm{v} / \mathrm{v})$ on a glass cover slide was evaporated over some days at $50^{\circ} \mathrm{C}$ and one day at $80^{\circ} \mathrm{C}$ and under vacuum.

AFM experiments were carried out with a Nanoscope II scanning probe microscope (Digital Instruments, Inc., Santa Barbara, CA, USA) at ambient conditions. Sample surfaces were scanned by a tiny pyramidal $\mathrm{Si}_{3} \mathrm{~N}_{4}$ tip which is attached to a microfabricated cantilever $(200 \mu \mathrm{m}$ triangular base). Deflections of the cantilever were registrated via deflection of a laser beam. Cantilevers with a force constant of 0.06 and 0.12 $\mathrm{N} / \mathrm{m}$ were applied. Scanning line frequencies were $1 \mathrm{~Hz}$ for large-scale scans and up to $39 \mathrm{~Hz}$ for smaller areas. Only low-pass filtering was used for large-scale images and in some cases filtering in Fourier space was applied for the atomic-scale images. For this purpose, the spots in the two-dimensional fast-Fourier-transformed pattern 2D-FFT (power spectrum), which correspond to the pronounced frequencies, were used for image reconstruction. Series of AFM images were collected in two different scanning directions, "up" and "down". Then averaged parameters were used for image characterization. In this way the influence of thermal drift is minimized. However, due to other reasons (non-linearity and creep of piezoelement, for example) the accuracy of AFM measurements was not higher than $10 \%$. 


\section{Results and discussion}

\subsection{Alkanes}

We have already shown that there is the possibility to record AFM images from the surfaces of platelet-type monocrystals of linear and cyclic alkanes [3]. Regular patterns were recorded which correspond to the packing of molecules in the crystal. Hills in the AFM map present individual methyl groups. Hydrogen and carbon atoms cannot be distinguished because of fast rotation of the methyl group around the $\alpha$-carbon-carbon bond. In addition to the periodic structure seen in our AFM images we also have observed defects (fig. 1). It is possible that the irregularly spaced surface holes in the periodical arrangement of AFM patterns are caused by surface defects (displacements of $n$-alkane chains from the surface). However, these dislocations were not stable in time; that leaves room for another explanation.

\subsection{Epitaxially crystallized polyethylene}

Arrays of oriented polyethylene molecules have been revealed in the AFM images of cold-extruded polyethylene [12]. In this material, poly- mer chains are aligned to the extrusion direction. The corresponding surface for AFM measurements had been prepared by ultramicrotomy cut along the extrusion direction. It is possible also to arrange polymer chains parallel to the surface by epitaxial crystallization of polyethylene on organic monocrystals. The surface of a polyethylene film, epitaxially crystallized on the (001) face of a monocrystal of anthracene, had been investigated. The polymer chain axis lies parallel to the substrate surface and the contact plane of the polymer with the substrate is (001). The largescale AFM image (fig. 2A) shows the well defined morphology associated with the orientation of crystallized material in two main directions with an angle of $\sim 70^{\circ}$ between them. This angle corresponds to the angle between (110) and (110) directions in the anthracene (001) plane. The atomic-scale AFM images have been obtained during the examination of individual lamellae. Though the quality of the presented image (fig. 2B) is not so good, the 2D-FFT pictures show the existence of linear AFM patterns (fig. 2C). The average distance of the chain-like structures is $0.48 \mathrm{~nm}$ which can be interpreted as distances between oriented polymer chains. The orientation of polymer chains with respect to the main lamellar direction is $\sim 85^{\circ}$. The obtained results,

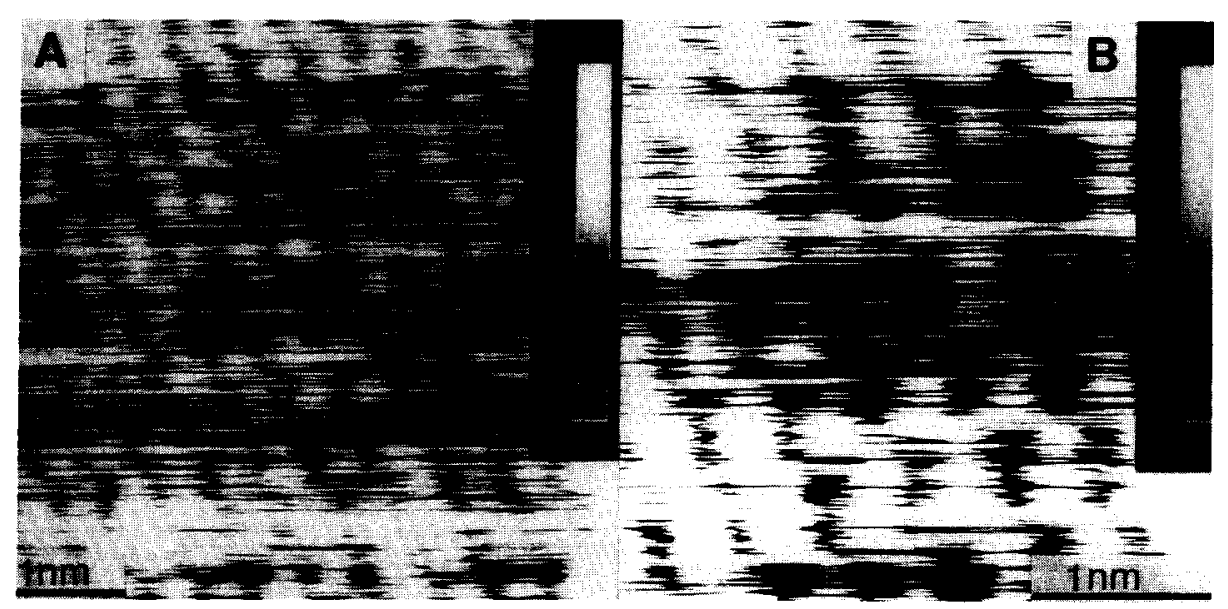

Fig. 1. Surface defects on the lamellar surface of $n$-alkane $\mathrm{C}_{33} \mathrm{H}_{68}(001)$ plane: (A) $5 \mathrm{~nm} \times 5 \mathrm{~nm}$, grey scale $0.5 \mathrm{~nm}$; (B) $3 \mathrm{~nm} \times 3$ $\mathrm{nm}$, grey scale $0.5 \mathrm{~nm}$. 


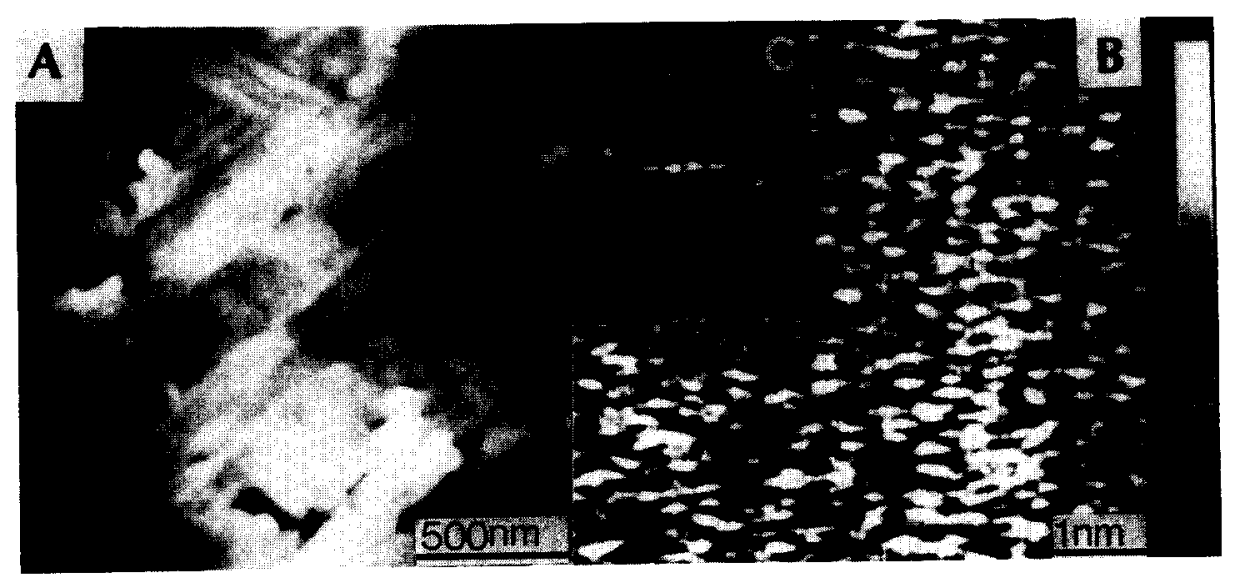

Fig. 2. AFM images of thin films of polyethylene (PE) epitaxially crystallized on anthracene (001): (A) morphology associated with the orientation of crystallized material in two main directions, $1800 \times 1800 \mathrm{~nm}$, grey scale $40 \mathrm{~nm}$; (B) chain-like structure of polyethylene, $7 \mathrm{~nm} \times 7 \mathrm{~nm}$, grey scale $0.3 \mathrm{~nm}$; (C) $2 \mathrm{D}$-FFT (power spectrum) of (B) before filtering.

concerning the orientation of lamellae and polymer chains, correspond to the conclusions derived from electron microscopy data [13]. It should be mentioned that organic crystals, which are actually used for epitaxial crystallization of polymers, have been already examined. The surface crystal structure of tetracene, the four-ring ana$\log$ in the naphthalene-anthracene series of linear, fused benzene rings, has been imaged with molecular resolution [14].

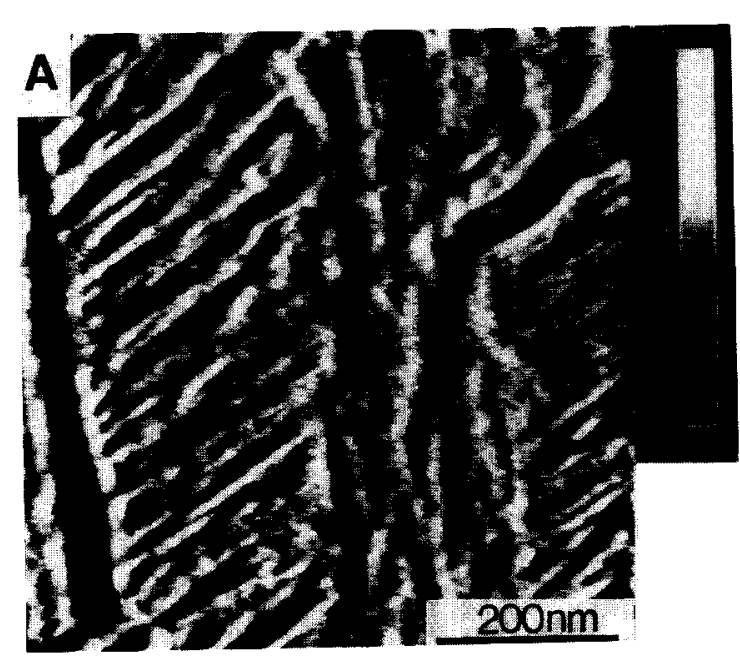

\subsection{Isotactic polypropylene}

We have shown the advantages of the AFM for morphology studies of the contact surface of thin films of $\alpha$-phase isotactic polypropylene, epitaxially crystallized on benzoic acid [7]. The AFM images reveal the lamellar structure as well as the methyl side group patterns in the exposed (010) planes.

The AFM large-scale views of iPP films, epi-

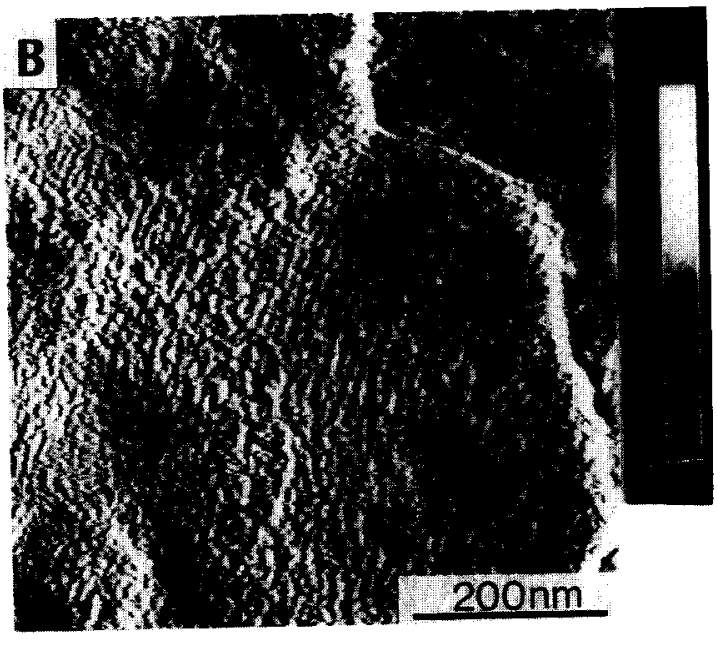

Fig. 3. AFM images of thin films of isotactic polypropylene epitaxially grown on different substrates: (A) morphology of the free surface of iPP, epitaxially grown on benzoic acid, $700 \mathrm{~nm} \times 700 \mathrm{~nm}$, grey scale $2 \mathrm{~nm}$; (B) morphology of the free surface of iPP, epitaxially grown on polyamide 11 (PA11 oriented by epitaxially crystallization), $600 \mathrm{~nm} \times 600 \mathrm{~nm}$, grey scale $0.1 \mathrm{~nm}$. 

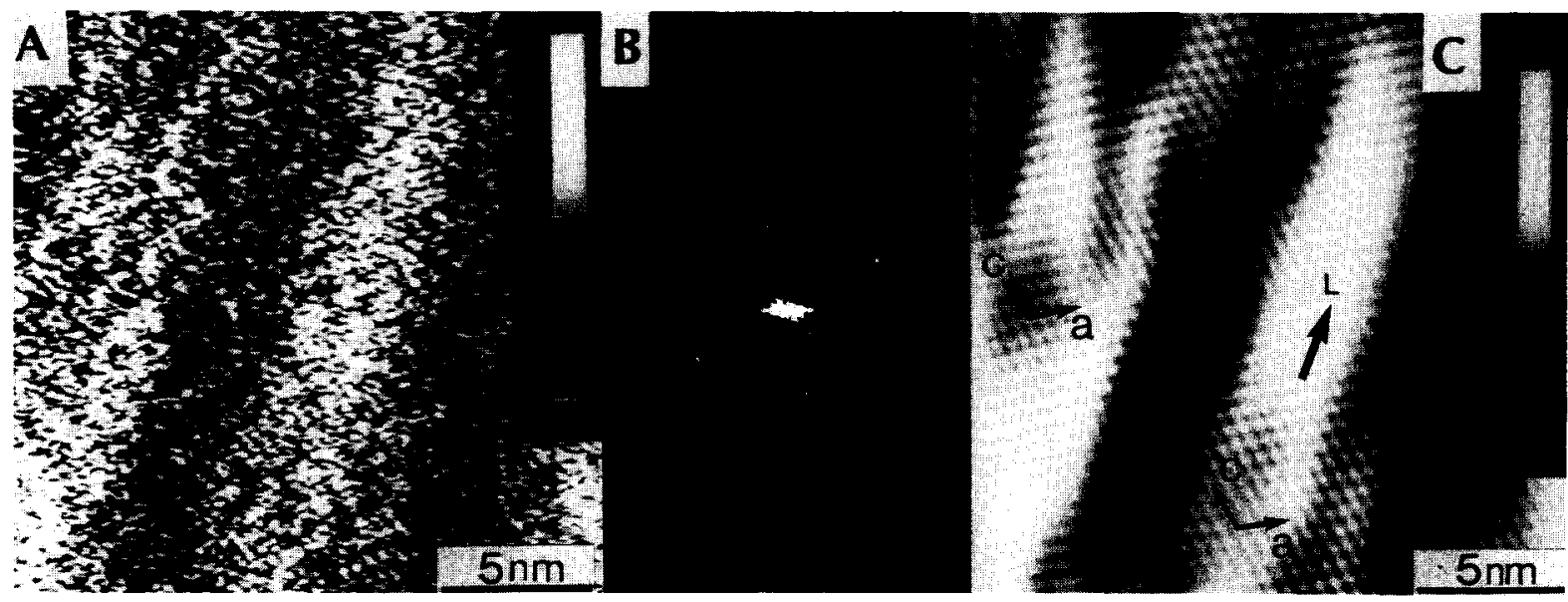

Fig. 4. AFM images of thin films of the $\gamma$-phase of iPP epitaxially grown on anthracene, contact surface (A) molecular structure, unprocessed image, $25 \mathrm{~nm} \times 25 \mathrm{~nm}$, grey scale $0.4 \mathrm{~nm}$; (B) 2D-FFT (power spectrum) of (A) before filtering; (C) molecular structure, processed using $2 \mathrm{D}-\mathrm{FFT}$ of (A), $25 \mathrm{~nm} \times 25 \mathrm{~nm}$, grey scale $0.4 \mathrm{~nm}$, the arrow $\mathrm{L}$ indicates the lamellar orientation, the arrow $\mathrm{c}$ indicates the chain orientation (helix axis orientation)

taxially crystallized on different substrates (i.e. benzoic acid, potassium salt of 4-chloro-benzoic acid or polyamide 11), show all the same well resolved and ordered large-scale views, fig. 3 . The brighter rows are separated equidistantly with a periodicity of $12.8 \mathrm{~nm}$. This periodicity corresponds well with the average lamellar thickness, determined by low-angle electron diffraction of gold-decorated films [15].
The molecular structure of the $(010)$ plane of the lamellae has been resolved in the atomic-scale image (figs. 4A and 4C). The appropriate 2D-FFT (fig. 4B) gives evidence for a triclinic lattice, with two main periodicities of $a_{\mathrm{AFM}}=0.69 \pm 0.05 \mathrm{~nm}$, $c_{\mathrm{AFM}}=0.66 \pm 0.05 \mathrm{~nm}$ and $\beta_{\mathrm{AFM}}=98^{\circ} \pm 3^{\circ}$. The repeat distances of the AFM images are close to the corresponding crystallographic parameters, $a_{\text {cryst }}=0.654 \mathrm{~nm}, c_{\text {cryst }}=0.650 \mathrm{~nm}, \beta_{\text {cryst }}=99.6^{\circ}$,

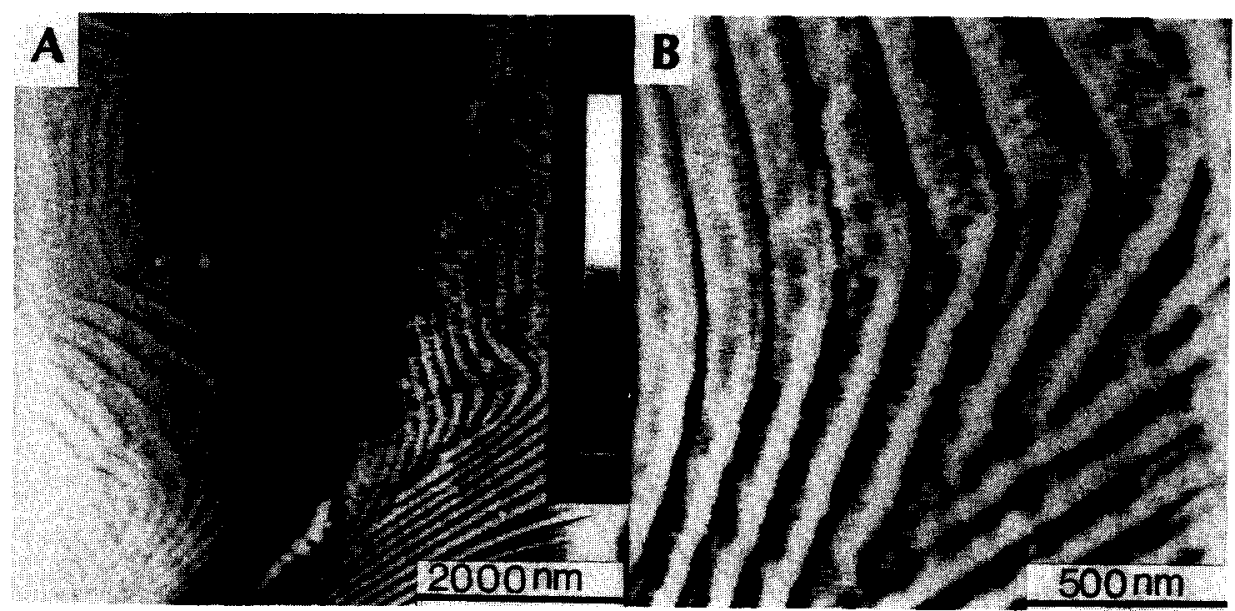

Fig. 5. Surface morphology of an ultrathin cut of polystyrene-b-poly(-2-vinylpyridine): (A) lamellar structure, $600 \mathrm{~nm} \times 600 \mathrm{~nm}$, grey scale $40 \mathrm{~nm}$; (B) $1500 \mathrm{~nm} \times 1500 \mathrm{~nm}$, grey scale $40 \mathrm{~nm}$. 
$\gamma_{\text {cryst }}=99^{\circ}$. The chain is tilted $50^{\circ}$ to the lamellar surface. This indicates that the observed structure is the triclinic $\gamma$-phase of iPP, and thus it is concluded that the helices we are looking at are left handed. It is known that $\gamma$-phase crystals have their chain axis inclined at $50^{\circ}$ to the lamellar surface [5]. The discrimination between two structural different contact surfaces in the $(010)$ layer becomes possible.

\subsection{Polystyrene-b-poly-(2-vinylpyridine) copoly- mers}

Also the surface morphology of a synthetic microphase-separated block copolymer, polystyrene-poly-(2-vinylpyridine) (PS $/ \mathrm{PVP}), M_{\mathrm{n}}$ $=40000$, weight ratio PS : PVP $=80: 20$, has been studied by AFM. Transmission electron microscopic studies (ESI) have been used to assign the somewhat broader stripes with a width of $\sim 50$ $\mathrm{nm}$ to the PS phase [16]. The absolute value for the lamellar width, however, depends strongly on the angle between the section plane and the lamella normal. With the lamella normal parallel to the section plane the width appears to be minimal whereas all other angles give bigger values. The morphology observed by AFM corresponds well with the results obtained by ESI. Fig. 5 presents the surface morphology of an ultrathin section. It clearly demonstrates the lamellar mor- phology with lamellar widths of $50-90 \mathrm{~nm}$. The PS/PVP films observed in figs. 5A and 5B are prepared by ultramicrotomy. It is known, that the morphology of block copolymers depends strongly on the method of preparation, i.e. solvent, temperature and rate of solvent evaporation [11].

A totally different film preparation is shown in figs. $6 \mathrm{~A}$ and $6 \mathrm{~B}$. A thin film of PS/PVP on a glass cover slide was imaged with AFM. After evaporation of the solvent (toluene/methanol $80: 20)$ round islands with the well ordered lamellar structures at the borders were observed.

\section{Conclusion}

The results presented above demonstrate the potential of atomic force microscopy for analysis of surface structure and morphology of polymeric materials and polymer-related model compounds. The force maps reveal lamellar surface orientation as well as the molecular structure of lamellae. AFM makes it possible to distinguish different crystal phases and even chain conformations on polymer surfaces. Given the resolution achieved, AFM becomes a significant technique for studies of polymer morphology, chain arrangement, secondary structure, conformation analysis, molecular disorder and crystal structure.

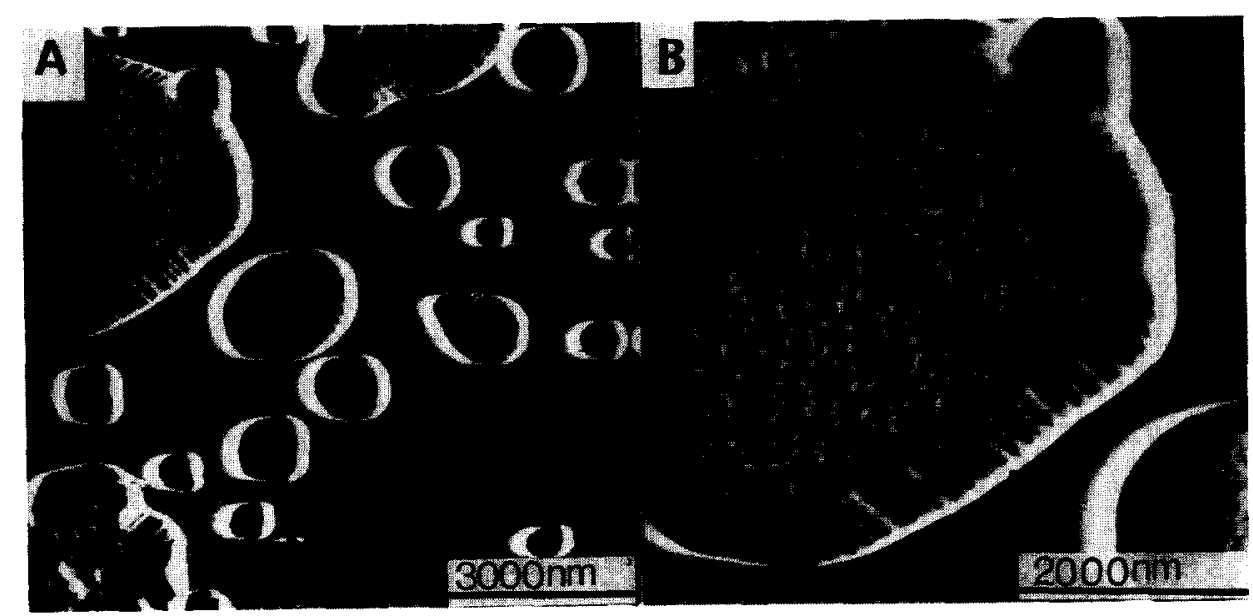

Fig. 6. Thin films of polystyrene-b-poly-(2-vinylpyridine) prepared on glass cover slides: (A) $9000 \mathrm{~nm} \times 9000 \mathrm{~nm}$, grey scale $40 \mathrm{~nm}$. ordered lamellar structures at the borders; (B) $5000 \mathrm{~nm} \times 5000 \mathrm{~nm}$, grey scale $40 \mathrm{~nm}$. 


\section{Acknowledgement}

W.S. thanks Fraunhofer Gesellschaft FhG-IPM for a grant. We thank cordially Dr. M. Kunz for the PS/PVP samples and for helpful discussions. To Eva Schill-Wendt we are grateful for her photographic work.

\section{References}

[1] J.C. Wittmann and B. Lotz, J. Polym. Sci. 23 (1985) 205.

[2] H. Drottloff, D. Emeis, R. Waldron and M. Möller, Polymer 28 (1987) 1200.

[3] W. Stocker, G. Bar, M. Kunz, M. Möller, S.N. Magonov and H.-J. Cantow, Polym. Bull. 26 (1991) 215.

[4] G. Natta and P. Corradini, Nuovo Chim. Suppl. 15 (1960) 40.

[5] B. Lotz, S. Graff and J.C. Wittmann, J. Polym. Sci. Polym. Phys. 24 (1986) 2017.
[6] B. Lotz and J.C. Wittmann, J. Polym. Sci. 24 (1986) 1541.

[7] B. Lotz, J.C. Wittmann, W. Stocker, S.N. Magonov and H.-J. Cantow, Polym. Bull. 26 (1991) 209.

[8] M. Ashida, Y. Ueda and T. Watanabe, J. Polym. Sci. Phys. 16 (1978) 179.

[9] J.C. Wittmann, A.M. Hodge and B. Lotz, J. Polym. Sci. Phys. 21 (1983) 2495.

[10] G. Strobl, B. Ewen, E.W. Fischer and W. Piesczek, J. Chem. Phys. 61 (1974) 5257.

[11] M. Kunz, M. Möller and H.-J. Cantow, Makromol. Chem. Rapid Commun. 8 (1987) 401.

[12] S.N. Magonov, K. Qvarnström, V. Elings and H.-J. Cantow, Polym. Bull. 25 (1991) 689.

[13] J.C. Wittmann and B. Lotz, J. Polym. Sci. Phys. Ed. 19 (1981) 1837.

[14] R.M. Overney, I. Howland, J. Frommer, E. Meyer and H.-J. Güntherodt, J. Chem. Phys. 942, 2 (1991) 8441.

[15] J.C. Wittmann and B. Lotz, Progr. Polym. Sci. 15 (1990) 900.

[16] M. Kunz, M. Möller, U.R. Heinrich and H.-J. Cantow, Macromol. Chem. Macromol. Symp. 23 (1989) 57. 